\title{
Color-selective holographic retroreflector array for sensing applications
}

\begin{abstract}
Rajib Ahmed ${ }^{1}$, Ali K Yetisen ${ }^{2,3}$, Seok Hyun Yun ${ }^{2,3}$ and Haider Butt ${ }^{1}$
Corner cube retroreflectors (CCRs) have applications in sensors, image processing, free space communication and wireless networks. The ability to construct low-loss wavelength filters embedded in CCRs can enable the development of wavelength multiplexing, tunable lasers and photonic integrated circuits. Here we created an $\sim 10-\mu \mathrm{m}$-thick holographic corner cube retroreflector (HCCR) array that acted as a color-selective wavelength filter and diffracted light at broad angles. Angle-resolved spectral measurements showed that the Bragg peak of the diffracted light from the HCCR array could be tuned from 460 to $545 \mathrm{~nm}$ by varying the incident angle. The HCCR array also exhibited a wavelength-selective tuning capability based on the rotation angle in the visible spectrum. HCCRs projected holographic images with the rotational property in the far field. The utility of the HCCR was demonstrated as optical temperature and relative humidity sensors that produced a visible colorimetric response for rapid diagnostics.
\end{abstract}

Light: Science \& Applications (2017) 6, e16214; doi:10.1038/lsa.2016.214; published online 24 February 2017

Keywords: Bragg gratings; corner cube retroreflectors; diffraction optics; holography; sensors

\section{INTRODUCTION}

Corner cube retroreflectors (CCRs) consist of three mutually perpendicular intersecting flat surfaces that directly reflect incident light back to its source ${ }^{1,2}$. The incident light is internally reflected three times in CCRs, and their directional feature is independent of the incident angle. CCRs have applications in satellite communication, laser components and antennas ${ }^{3-5}$. In particular, the phase conjugation property of a CCR array has been widely used for wavefront correction and for enhancing the resolution in image processing ${ }^{6,7}$. The phase conjugation property of CCR array surfaces can also be utilized in wavefront sensing, phase-conjugated interferometry and Fourier transform holography ${ }^{8-10}$. Recently, microelectromechanical retroreflectors have been fabricated for miniaturized applications ${ }^{11,12}$. CCRs fabrications are typically based on micromechanical processing or photolithography ${ }^{13-16}$. These fabrication approaches are costly, expertise dependent and time-consuming, thus limiting their scalability for practical applications in photonics.

Here we show the development of a three-dimensional HCCR array using Denisyuk reflection recording. The HCCR array does not exhibit all the analogous optical properties when compared with CCRs. However, the HCCR array is planar and thinner, functions as a narrow-band wavelength filter and exhibits the rotational property. In this work, we analyze the directional reflection properties of the CCR array through numerical modeling; their holographic images are recorded using silver halide chemistry. We also present a unifying view regarding the rotational property of HCCRs. Angle- resolved reflection measurements of the HCCRs show a reasonable diffraction efficiency (DE), along with the directional color filtering of broadband light. Finally, we demonstrate the utility of HCCR in colorimetric temperature and relative humidity $(\mathrm{RH})$ sensing. This work is the first report of the production of HCCR arrays, which may have potential applications in compact optical systems and sensors.

\section{MATERIALS AND METHODS}

Holographic retroreflector fabrication

The HCCR array was fabricated using Denisyuk reflection holography. A HeNe laser beam $(\lambda=632.8 \mathrm{~nm}, 20 \mathrm{~mW})$ was passed through a beam expander $(\varnothing=6 \mathrm{~cm})$ and a collimator. In Denisyuk reflection mode, an image of an array of CCRs (object) was recorded in a light-sensitive emulsion (Figure 1a). The holographic plates $(2 \mathrm{~cm} \times 2 \mathrm{~cm})$ consisted of a gelatin emulsion containing light-sensitive silver bromide (AgBr) nanocrystals (NCs) $(\varnothing=10-20 \mathrm{~nm})$ conjugated to quinaldine blue $\left(1,1^{\prime}-\right.$ diethyl-2,2'-carbocyanine) dye. The sample was exposed to a laser beam after being tilted $\left(\theta \leq 5^{\circ}\right)$ from the mirror surface plane. The tilt angle created a slanted interference pattern in the recording medium, which separated the diffracted light from the specular reflection (off the glass substrate). Low tilt angles reduced the effect of the transmission grating and increased the $\mathrm{DE}^{17}$. An index matching fluid was used to reduce the transmission grating effect. The interference pattern formed in the holographic plate involved

${ }^{1}$ Microengineering and Nanotechnology Laboratory, School of Engineering, University of Birmingham, Birmingham B15 2TT, UK; ${ }^{2}$ Harvard Medical School and Wellman Center for Photomedicine, Massachusetts General Hospital, Cambridge, MA 02139, USA and ${ }^{3}$ Harvard-MIT Division of Health Sciences and Technology, Massachusetts Institute of Technology, Cambridge, MA 02139, USA

Correspondence: H Butt, Email: h.butt@bham.ac.uk

Received 6 January 2016; revised 12 August 2016; accepted 1 September 2016; accepted article preview online 5 September 2016 

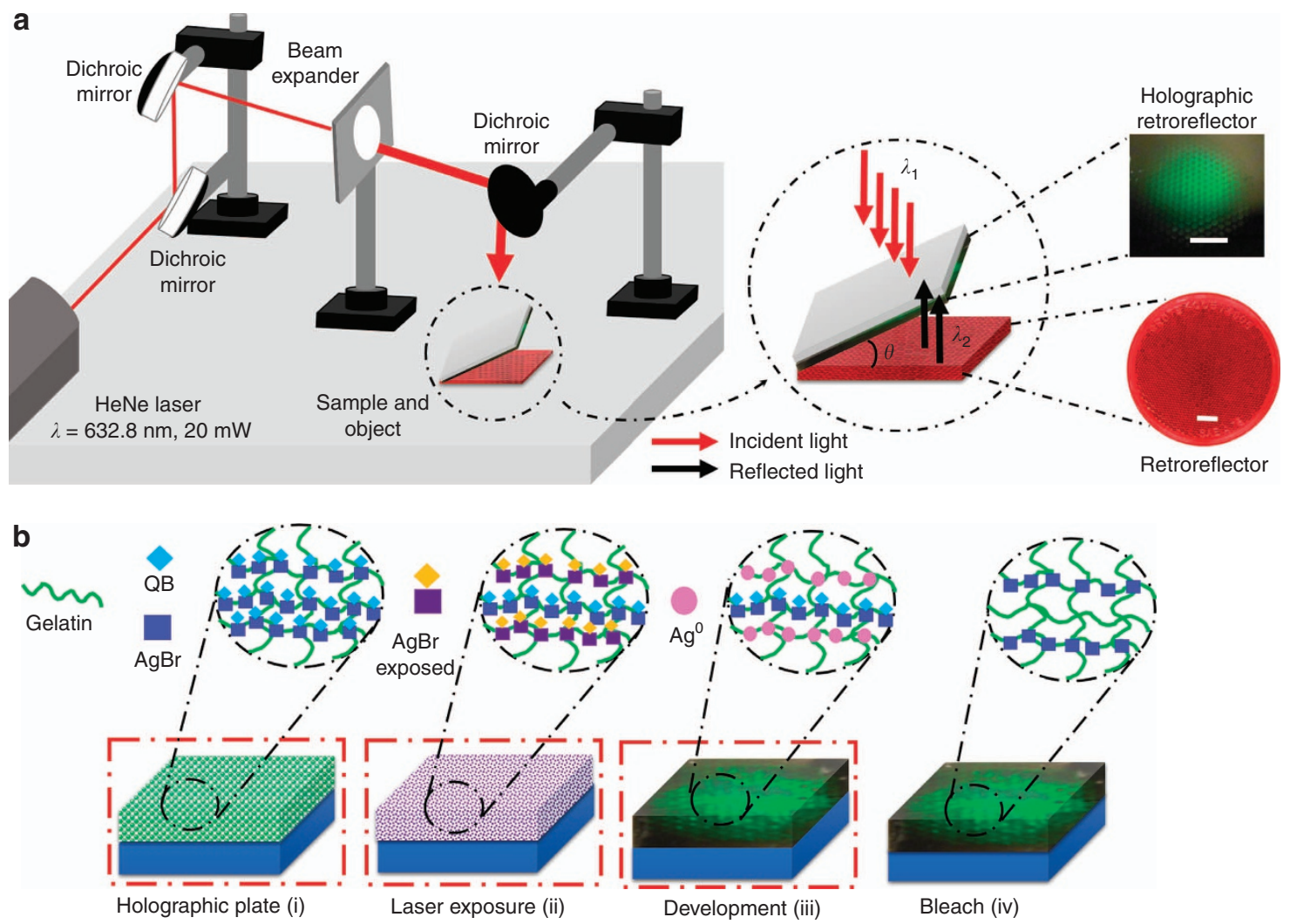

C
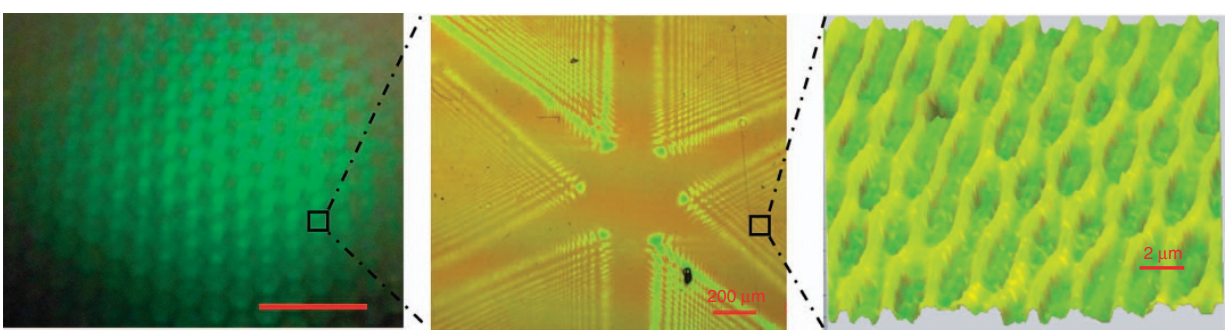

Figure 1 Holographic recording of a HCCR array in light-sensitive media. (a) The experimental setup in Denisyuk reflection mode. Scale bars $=1 \mathrm{~cm}$. (b) Holographic retroreflector array preparation: (i) using a gelatin matrix containing photosensitized $\mathrm{AgBr} \mathrm{NCs}$, photosensitization was performed in the presence of quinaldine blue (QB); (ii) gelatin matrix with AgBr NCs was exposed to a laser beam $(632.8 \mathrm{~nm})$ to record an image of a retroreflector array; (iii) photographic developer was used to reduce the exposed $\mathrm{AgBr} N C s$ to silver metal ( $\mathrm{Ag}$ ) NPs; (iv) the hologram was bleached to increase $\mathrm{DE}$, and $\mathrm{Ag}^{0} \mathrm{NPs}$ were converted back to $\mathrm{AgBr} \mathrm{NCs}$. (i-iv) were performed under green safe lighting. (c) Top view of the holographic retroreflector and its surface geometry, consisting of triangular sections on the surface and an internal multilayer structure. Scale bar $=1 \mathrm{~cm}$.

two beams: incident (reference) beam, $\lambda_{1}$, and reflected (object) beam, $\lambda_{2}$. A corner cube reflector array (the dimension of each CCR is $\sim 0.2 \mathrm{~cm}$ ) was used as an object to record the interference pattern within the gelatin matrix.

The sample development was based on silver halide chemistry (Figure $1 \mathrm{~b})^{18,19}$. The gelatin contained $\mathrm{AgBr} \mathrm{NCs}$ that had been optimized for high-resolution holography. Glass was used as a substrate, and its surface was functionalized using (3-aminopropyl)triethoxysilane in acetone $(1: 100, \mathrm{v} / \mathrm{v})$. Gelatin is a transparent medium and has flexible pores that can accommodate AgBr NCs and $\mathrm{Ag}^{0}$ nanoparticles (NPs). The gelatin holographic plates were preswollen by immersing them in a bath of aqueous solution of triethanolamine $(10 \%, \mathrm{v} / \mathrm{v})$ for $1 \mathrm{~min}$ and then dried in a cold airflow at $60 \% \mathrm{RH}$ for $1 \mathrm{~h}$. Upon exposure to laser light, a latent image was formed in the emulsion through the disruption of the AgBr ion-pair with a laser light, that is, $\mathrm{AgBr}+\mathrm{h} \gamma \rightarrow \mathrm{Ag}^{+}+\mathrm{Br}^{-}+\mathrm{e}^{-}$, and subsequent silver atom formation at an electron trap site in the $\mathrm{AgBr} \mathrm{NC}$, that is, $\mathrm{Ag}^{+}+\mathrm{e}^{-} \rightarrow \mathrm{Ag}^{020}$. A photographic developer containing 4-(methylamino)phenol hemisulfate $(\mathrm{pH} \sim 12)$ was used to amplify the latent image in the $\mathrm{AgBr} \mathrm{NCs}$ into silver metal $\left(\mathrm{Ag}^{0}\right) \mathrm{NPs}$ to form a multilayer structure of the visual image. Bright fringes (antinodes) in the standing wave were amplified as $\mathrm{Ag}^{0} \mathrm{NPs}$. The emulsion was bleached $\left(\mathrm{KBr}\right.$ and $\left.\mathrm{CuSO}_{4}\right)$ by converting the $\mathrm{Ag}^{0}$ NPs back to transparent $\mathrm{AgBr}$ NCs. Bleaching increases the DE and reduces light scattering ${ }^{18,21}$. After drying, the HCCR exhibited efficient green diffraction, and the surface contained micropatterned arrays (Figure 1c). The diffraction from the HCCR effect was due to the periodic arrangement of $\mathrm{AgBr}$ NCs within the gelatin matrix, obeying Bragg's law, and the surface structures contributed to angular anisotropy. 
Computational modeling, phase conjugation and rotational property of CCR array

To understand the optical interference patterns, which produce the HCCRs, optical simulations of an array of CCRs were performed (Supplementary Information, Supplementary Fig. S2). The reflection and directional properties of the CCRs arrays were analyzed using the finite element method. The structure of the retroreflector was considered as a two-dimensional triangular grating (Supplementary Information, Supplementary Fig. S2). To obtain approximation results, the triangular grating's spacing and depth were considered to

$\sim 15-20$ times larger than the incident wavelength, $\lambda(d \gg>\lambda)$. For simplicity, a two-dimensional simulation was performed using triangular meshes (the maximum mesh size was approximately one-tenth of the input wavelength) with a fine domain structure, for a total of 23 698 meshing elements ${ }^{22,23}$.

The phase conjugation and rotation property of a CCRs/HCCR array are based on wavefront analyses of incident and reflected/ diffracted light. Consider an arbitrary incident wave under paraxial approximation $\left(K_{z}=K=\frac{2 \pi}{\lambda}\right)$ :

$$
E_{i}(x, y, z)=A(x, y) \exp (i \varphi(x, y)) \exp (-i k z)
$$

The reflected light from the CCRs array is

$$
\begin{aligned}
E_{\mathrm{CC} \text { Array }}(x, y, z)= & \sum_{m, n} A(2 m h-x, 2 n h-y) \exp (i \varphi(2 m h-x, 2 n h-y)) \\
& \exp (+i k z) a_{\mathrm{CC}}(x, y) \operatorname{rect}\left(\frac{x-m h}{h}, \frac{y-n h}{h}\right)
\end{aligned}
$$

where $\operatorname{rect}(x, y)$ is a rectangular function equal to 1 , where $\operatorname{abs}(x, y)$ $<1 / 2$, and is 0 otherwise; and $a_{\mathrm{cc}}(x, y)$ is a scalar quantity known as the aperture function and indicates the reflected beam amplitude (Supplementary Information 'Directional, Phase Conjugation and Rotational Property of CCR Array'). The wavefront reflected from the CCR array surface showed a reversed phase but the same amplitude sign as that of the incident wavefront. This might have been due to the triangular surface of the CCRs array (side view), where the reflected wavefront was disrupted, cut into segments and shifted along the direction of propagation, showing analogous properties to those of diffraction gratings and Fresnel lenses ${ }^{6}$. The rotation property of CCRs, based on wavefront analyses of incident and reflected light, was also studied (Supplementary Information 'Directional, Phase Conjugation and Rotational Property of CCR Array'). If the incident holographic image rotates in the clockwise direction, the far-field projected image from CCR and HCCR rotates in the reverse direction (Supplementary Information, Supplementary Fig. S2 and Supplementary Movie 1). The rotational property of the HCCR array was based on light diffraction from the HCCR surface. If the sample rotated (for a broadband light source) in the clockwise direction, the diffracted color light from the HCCR array rotated in the anticlockwise direction (Supplementary Information, Supplementary Fig. S2 and Supplementary Movie 2). The rotational property was found to be valid for the CCR and HCCR retroreflector.

\section{RESULTS AND DISCUSSION}

\section{Optical characterization}

The HCCR arrays were spectroscopically characterized in reflection mode. The sample was illuminated using a broadband light source (450-1100 nm). An Ocean Optics 2000 spectrometer (450-1100 nm, $0.2 \mathrm{~nm}$ resolution) was used to measure the reflected and diffracted light. A goniometer setup was utilized to obtain spectra at different incident angles to capture the maximum reflection and diffraction peaks (Figure 2a). The reflection readout of the holographic retroreflector was found to be highly dependent on the incident angles (Figure 2b). In response to incident light, an intense broadband reflection signal (specular reflection from the substrate) was detected, as was narrow-band diffraction. The properties of the diffracted light were dependent on the incident angles and sample position.

The reflected broadband signal also displayed maximum peaks (Figure 2b). The peak blueshifted linearly as the incident angle was increased from the normal. This peak might represent the resonance of $\mathrm{Ag}^{0} \mathrm{NPs}$ within the gelatin. In addition, the narrow-band diffracted light was analyzed by varying the incident angles and moving the spectrophotometer probe to measure the maximum intensity (Figure 2c). The holographic retroreflector acted as a sensitive color filter by separating colors from the incident white light, depending on the incident angles. For the reflected white light (zero order), the incident and reflection angles were equal. However, for the diffracted narrow-band light (higher orders), the incident angles were different from the collection/diffraction angles, and the diffraction angles increased nonlinearly for larger incident angles. This was due to the volumetric multilayer structure in the HCCR array ${ }^{17}$. The separation between the reflected and diffracted light was $\sim 10^{\circ}$. However, this separation increased with larger incident angles from $12^{\circ}$ to $25^{\circ}$.

The reflected spectra mainly showed broadband light, with peaks in the range of 506-562 $\mathrm{nm}$ (green) and 478-497 nm (blue) for incident angles between $15^{\circ}$ and $65^{\circ}$. The peak that was originally at $562 \mathrm{~nm}$ $\left(i=15^{\circ}\right)$ shifted linearly from 558 to $478 \mathrm{~nm}$ for $i=20^{\circ}-65^{\circ}$, in $5^{\circ}$ increments, respectively. For larger incident angles, lower-intensity peaks were measured, which could have been due to the decreasing diffraction intensity. The HCCR array showed 20-40 nm lower Bragg peaks because the lattice spacing of the multilayer grating contracts due to bleaching and exposure in a swollen state. Figure $2 \mathrm{c}$ shows the normalized diffracted light spectra as the incident angle was changed. The peak blueshifted, and its intensity decreased at broad incident angles. Diffraction also showed a linear trend at different incident angles. The diffracted light's peak wavelengths $\left(\lambda_{\mathrm{p}}\right)$ were measured at different receiver positions, $\theta_{\mathrm{r}}(=r)$ (Figure $2 \mathrm{~d}$ ). The diffracted light shifted from green to blue and then to violet as the incident angle was increased. The shift to shorter wavelengths was due to the venetian blind effect $^{24}$. At broad incident angles, the lattice spacing seen by the white light may have been smaller compared with that at lower incident angles. The intensity of the diffracted light was low for angles $>65^{\circ}$.

Spectral measurements were performed using fixed incident angles $\left(i \leq 50^{\circ}\right.$ or $\left.\geq 50^{\circ}\right)$ and rotated positions. The Bragg peaks of the diffracted light changed as the sample was rotated. With anticlockwise rotation of the HCCR array, diffracted light rotated in the clockwise direction (Figure $3 \mathrm{a}$ and $3 \mathrm{~b}$ ). This occurred for the diffracted green light; the reflected white light remained in the same position $\left(i \leq 50^{\circ}\right)$. In retroreflectors, white light also rotates in this position. Similar results were also found with higher incident angle $\left(i \geq 50^{\circ}\right)$, the clockwise rotation of the HCCR array, where diffracted light rotated in the anticlockwise direction (Figure $3 \mathrm{c}$ and $3 \mathrm{~d}$ ). This rotation property was due to the tilt of the multilayer grating recorded within the gelatin matrix. The corner cube multilayer structure splits the wavefront and reverses the phase at every point in the reflected wave (Equation 2). As the HCCR array was rotated, the grating diffracted light at different collection angles. The HCCR array filters and diffracts the green color for incident angles less than $50^{\circ}$. However, it also diffracted different color combinations (green, blue, violet and their mix colors) for incidence angles $>50^{\circ}$ (Figure $3 \mathrm{~d}$ ). This is of interest as the HCCR 

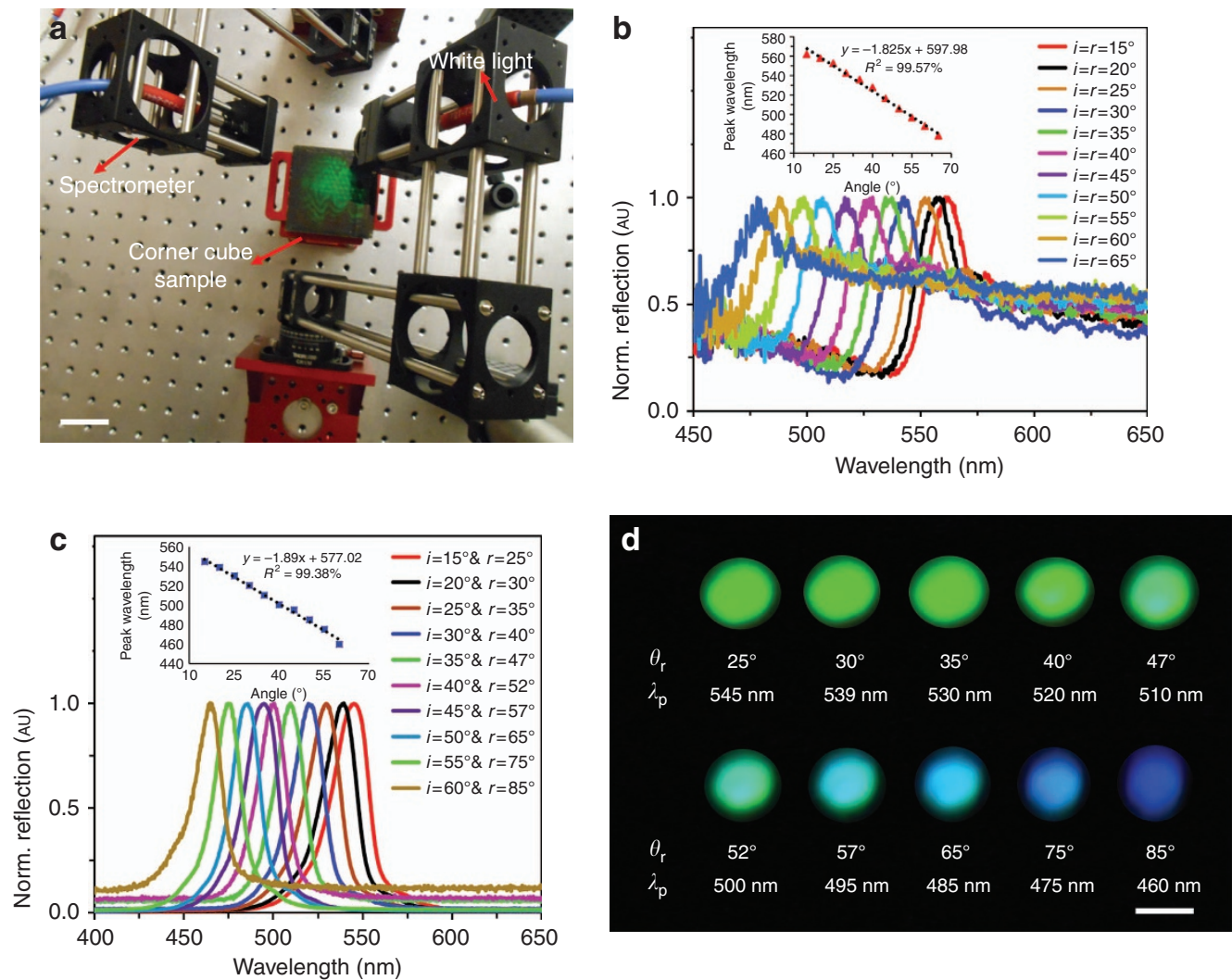

Figure 2 Angle-resolved measurements of the HCCR array. (a) Goniometer setup used to measure reflected light from the retroreflector under white light illumination. Scale bar $=6 \mathrm{~cm}$. (b) Normalized reflected light as a function of incident angles $\left(i=15^{\circ}-65^{\circ}\right)$; inset shows linear fitting of reflected peak wavelength as a function of incident angles. (c) Normalized monochromatic diffracted light as a function of incident angles $\left(i=15^{\circ}-60^{\circ}\right)$; inset shows linear fitting of diffracted peak wavelength as a function of incident angle. (d) Diffracted colors at different receiver positions $\theta_{\mathrm{r}}(=r)$ and peak wavelengths $\left(\lambda_{\mathrm{p}}\right)$. Scale bar $=2 \mathrm{~cm}$

array can be used as a tunable optical filter to select colors depending on the incident and azimuthal angles.

Figure $3 \mathrm{e}$ shows the experimental setup used to characterize the rotational property of the HCCR array. A red laser beam $(632 \mathrm{~nm}$, $20 \mathrm{~mW}$ ) was holographically shaped to form an image of an ant and was irradiated onto the HCCR array. A slight incident angle was chosen to record the reflected image separately. The reflected holographic image was projected onto a white screen with a centered perforation $(\varnothing=10 \mathrm{~mm})$. The reflected zero-order beam passed through the opening, and the holographic phase conjugated image (ant) was captured on the screen. Figure $3 \mathrm{f}$ shows the incident holographic image (left side) from a laser beam and reflected image (right side). The holographic projection also obeyed the rotational property: upon the clockwise rotation of the incident image, the reflected image also rotated anticlockwise (Supplementary Information, Supplementary Movie 1). The rotational property of the ant-hologram was also found to be valid with a single CCR/HCCR and to work properly with other visible light sources (for example, blue and green).

As the HCCR array diffracted various colors at different positions, an angle-dependent Fraunhofer diffraction analysis was performed ${ }^{25,26}$. The far-field diffraction patterns produced by the HCCR array in response to red, green and blue laser beams were studied. Figure $4 \mathrm{a}$ shows the experimental setup: the HCCR array was placed vertically on top of a rotation stage, equidistant from the laser source and the screen $(\sim 40 \mathrm{~cm})$. The incident angle of the laser beam was varied from $0^{\circ}$ to $60^{\circ}$ by rotating the HCCR array. The diffracted light was projected onto a semi-transparent screen. Figure $4 \mathrm{~b}-4 \mathrm{e}$ shows far-field diffraction patterns. The distance between the first-order and zeroorder decreased as the incident beam shifted to shorter wavelengths from red to blue, thus obeying Bragg's law.

At low incident angles, a highly intense green diffraction pattern was produced compared with the red and blue light. This was due to the overlap between the Bragg resonance of the HCCR array and the incident green laser beam. However, as the incident angle was increased, the blue diffraction pattern increased in intensity, which agreed with the results in Figure $2 \mathrm{c}$ and $2 \mathrm{~d}$, showing a blueshift with an increasing incidence angle. This capability showed that the HCCR arrays worked on the basis of diffraction, producing various orders compared to the conventional reflective CCRs and displaying an angular wavelength-selective image projection.

Angular dependent diffraction measurements of the HCCR were studied in response to red, green and blue laser light at rotational angles from $-90^{\circ}$ to $+90^{\circ}$ (Figure $4 \mathrm{f}-4 \mathrm{i}$ ). The sample holder and light source were supported by a stepper motor, which was rotated horizontally by $360^{\circ}\left(1^{\circ}\right.$ step size). An optical power meter was placed normally to the rotational stage to measure the diffraction power. Figure $4 \mathrm{f}-4 \mathrm{i}$ shows the $\mathrm{DE}$ and power as a function of the rotation angles. Maximum power and efficiency was measured using a red laser at the non-diffracted zero order. Symmetrical first-order diffraction peaks were observed on each side of the zero-order. The DE was measured as $\left(P_{\text {diff }} / P_{\text {in }}\right) \times 100 \%$, that is, the ratio of the diffracted power 

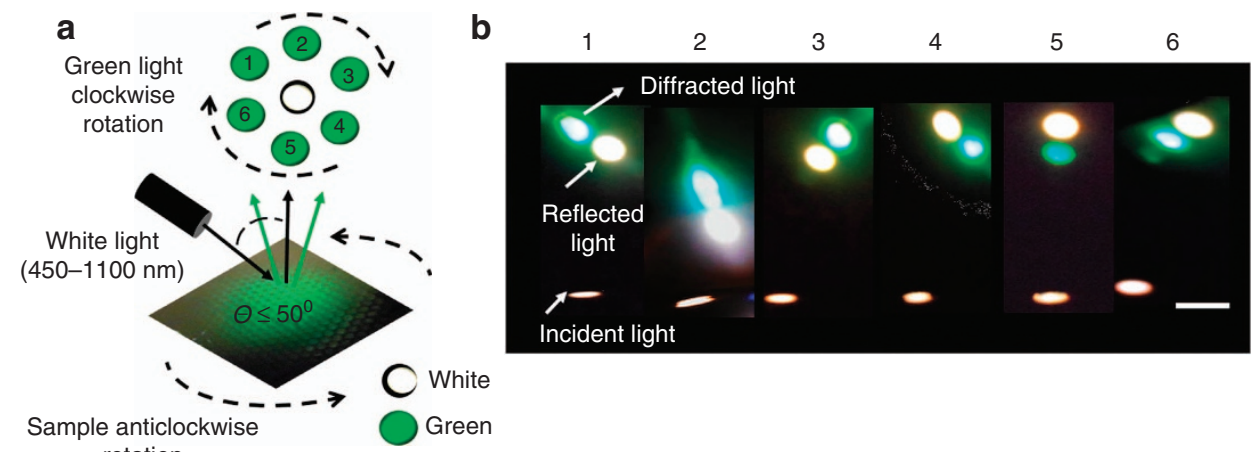
rotation

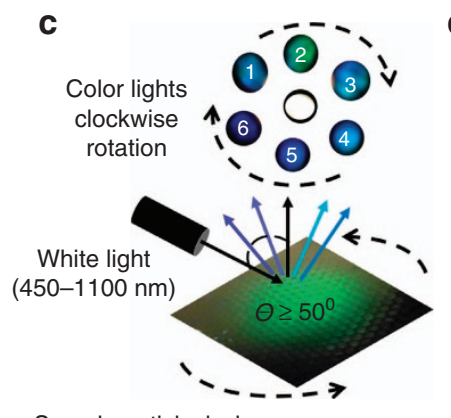

d

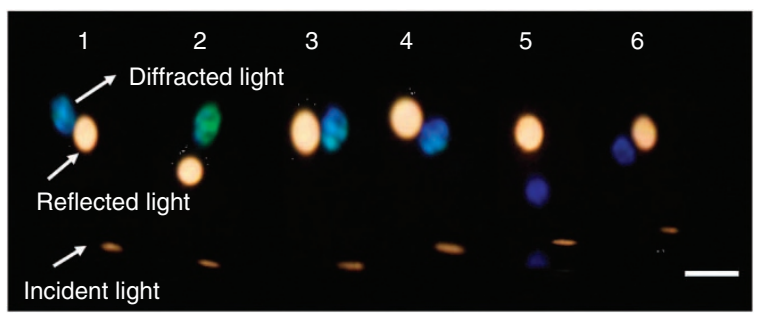

Sample anticlockwise
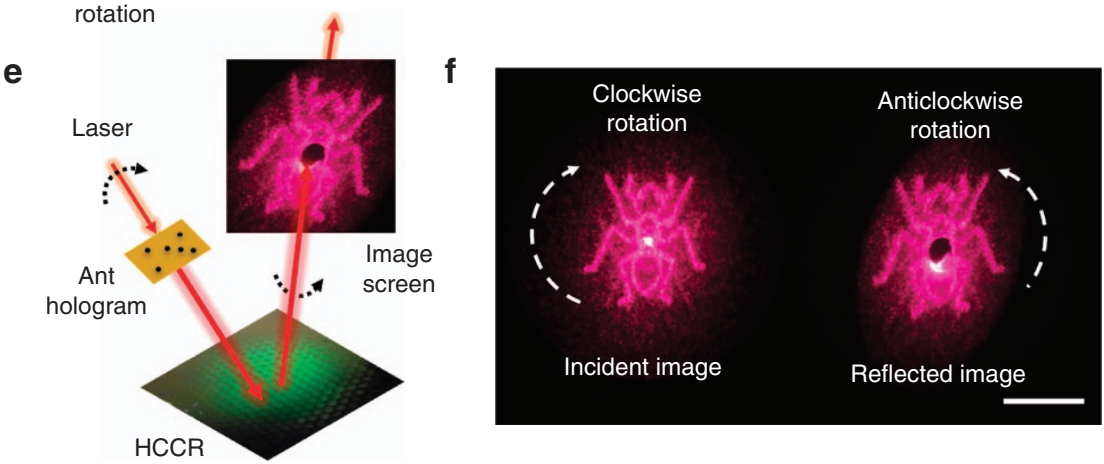

Figure 3 Rotational and color filtering characteristics of the HCCR array. (a) Incident light, specular reflection and diffracted green light. (b) With $i \leq 50^{\circ}$, clockwise rotation of the sample, green diffraction spot rotated anticlockwise. Scale bar $=2 \mathrm{~cm}$. (c) Incident light, specular reflection and diffracted narrowband light (various colors). (d) Narrow-band diffraction variation, with sample rotation at incident angles $i \geq 50^{\circ}$. Scale bar $=2 \mathrm{~cm}$. Supplementary Information (Supplementary Movie 2) shows the variation of the Bragg diffraction as the sample is rotated. (e) Experimental setup to analyze rotational property of the HCCR array. (f) Holographic incident image (left) from a laser beam and resultant projected image on a screen (right). Scale bar $=2 \mathrm{~cm}$.

$\left(P_{\text {diff }}\right)$ and incident power $\left(P_{\text {in }}\right)^{27-29}$. The average diffraction power and efficiency of the 1st-order peaks contributed approximately $8 \%$ and $4 \%$, respectively, to the overall diffraction power and efficiency for the red laser compared with the non-diffracted zero-order. Similarly, the average diffraction power and efficiency of the first-order green and blue peaks contributed $5 \%, 4 \%$ and $6.6 \%, 7 \%$, respectively. Moreover, the average diffraction power of the first-order peaks reduced to $50 \%$ and $80 \%$ for the green and blue laser, respectively, compared with the first-order red diffraction (Figure 4i). The DE and the diffracted power were reduced with shorter incident beam wavelengths. Similarly, the angular spread of the non-diffracted spot at the $3 \mathrm{~dB}$ point decreased as the wavelength increased (inset in Figure $4 \mathrm{f}-4 \mathrm{~h}$ ). This might have occurred because the spectral range of the gelatin matrix containing the $\mathrm{AgBr}$ emulsion was $580-650 \mathrm{~nm}$ and the sample was recorded using a red $\operatorname{laser}^{30}$. Power fluctuations were also measured between the symmetrical peaks (Figure 4i). Distortion at lower order diffracted peaks was also found in the literature ${ }^{31}$. The
HCCR showed a lower diffraction efficiency compared with the normal CCR array, but it presented the added advantage of being flat and compact $(\sim 10 \mu \mathrm{m})$. The optical property of the HCCR is similar to that of the other diffractive optical elements (for example, Bragg grating) and shows less intense diffraction ${ }^{6,29}$. The first-order diffracted red, green and blue light showed almost the same distance $\left(6^{\circ}\right)$ from the zero-order. The difference was visible in the far field (Figure $4 \mathrm{~b}-4 \mathrm{e}$ ). The resolution of the HCCR was measured as $5052 \mathrm{lp} \mathrm{mm}^{-1}$, with a maximum $\mathrm{NC}$ grain size of $\sim 20 \mathrm{~nm}$. The resolution of the recorded hologram was calculated as $\gamma=(2 n \sin (\delta / 2)) / \lambda$, where $\gamma$ is the spatial frequency, $n$ is the refractive index of the emulation (1.6), $\lambda$ is the recording wavelength $(632.8 \mathrm{~nm})$ and $\delta$ is the angle between the object and reference beam $\left(175^{\circ}\right)$. In the present work, visible light diffraction and broadband light were chosen because they were readily available and suitable for visual detection by the eye. The diffraction property can be extended to the ultraviolet (UV) and near-infrared (IR) region of the spectrum. For 


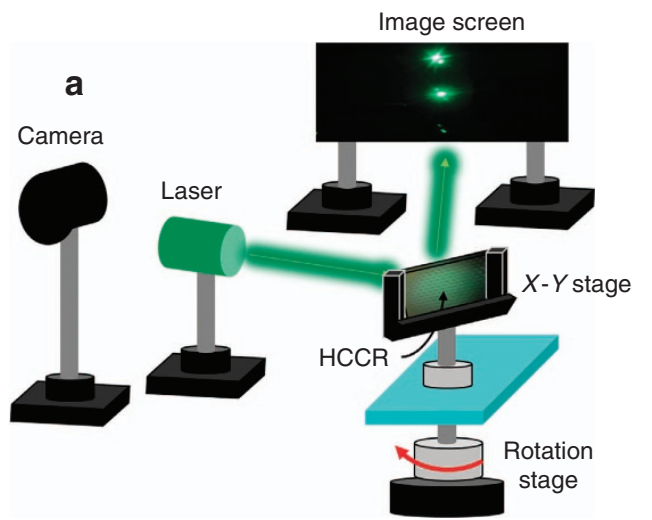

d
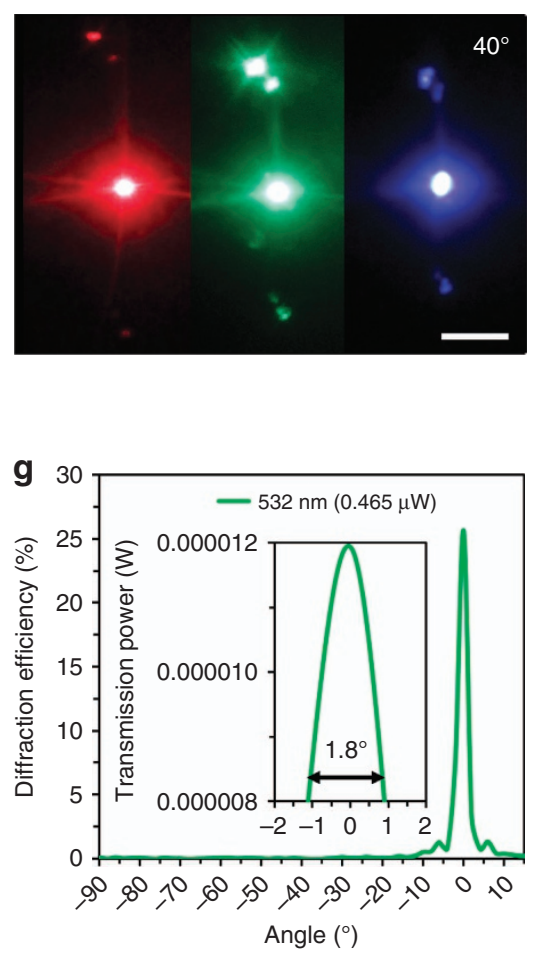

b

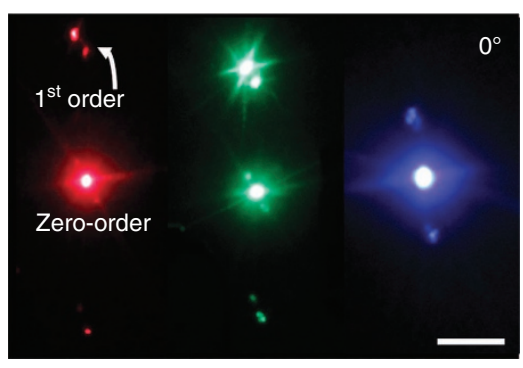

e
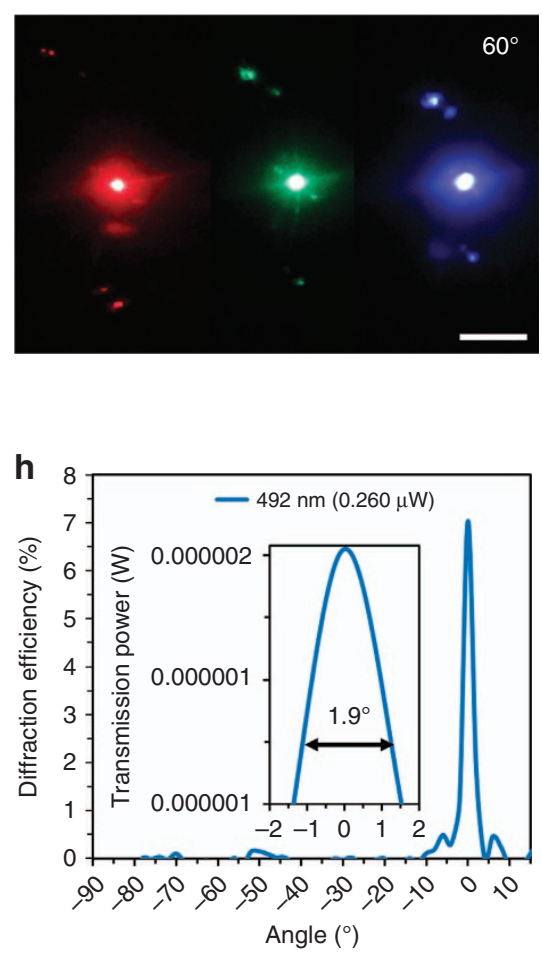

C

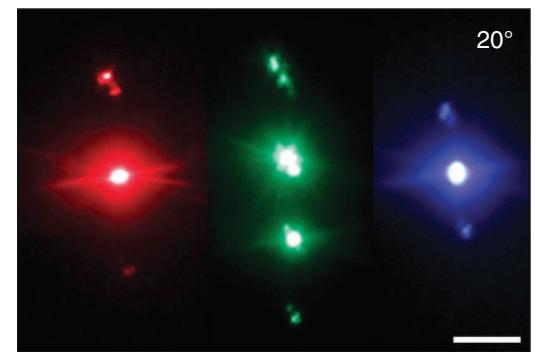

f

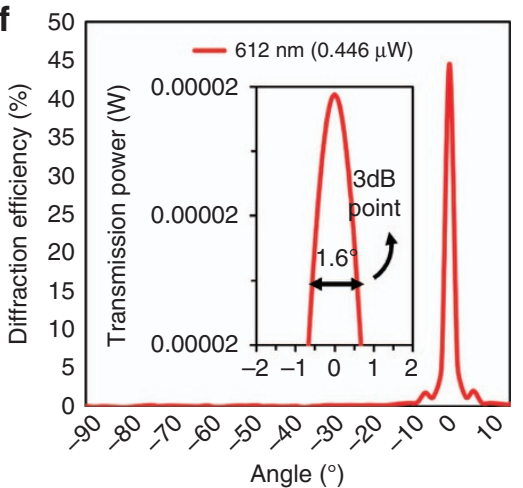

i

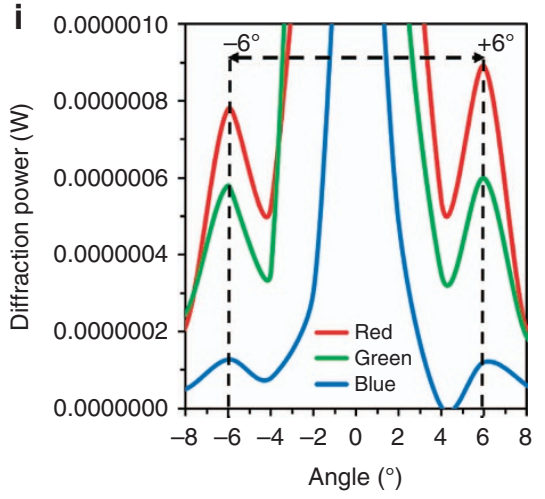

Figure 4 Characterization of visible diffraction from the HCCR array. (a) Experimental setup used for shining laser light on the HCCR array at various angles and projecting the diffracted light on a screen. (b-e) The diffraction patterns in response to red, green and blue laser light, irradiated at incidence angles from $0^{\circ}$ to $60^{\circ}$. Scale bars $=2 \mathrm{~cm}$. ( $\left.\mathbf{f}-\mathbf{i}\right)$ Angular DE measurement of the HCCR. The DE and intensity in response to red, green and blue laser light at rotation angles from $-90^{\circ}$ to $+90^{\circ}$. The $\mathrm{DE}$ and intensity decrease as wavelength increases; however, the angular spread of the non-diffracted spot at the $3 \mathrm{~dB}$ point increases.

this, the hologram needs to be recorded at shorter or longer wavelengths using UV or IR wavelengths, where the diffraction peak of the multilayer gratings is limited by Bragg's law. In addition, to tune the Bragg peak, pseudo-color holograms can be produced by preswelling or shrinking the recording media.

\section{Temperature and RH sensing}

To demonstrate the utility of the HCCR, we show an application in sensing. Figure 5a shows the conceptual diagram of the HCCR temperature and RH sensors. The Bragg peak of the diffracted color light $\left(\alpha_{\mathrm{d}}\right)$ and reflected white light $\left(\alpha_{\mathrm{r}}\right)$ from AgBr NC multilayers of the holographic polymer film responds to variation in temperature and $\mathrm{RH}$. The sensing principle is based on $\mathrm{AgBr} \mathrm{NC}$ multilayer structures that can swell or shrink in response to external stimuli. The grating structure acts as an optical transducer and can quantify the external stimuli by shifting the Bragg peak in the visible spectrum. The HCCR sensor responds to the incident white light and diffracted green (G) light at the steady-state condition (no stimuli); it acts as a reference between transition conditions such as swelling or shrinking period due to external stimuli. At high temperature and lower RH values, the lattice spacing of the internal multilayer shrinks, which shifts the Bragg peak to shorter wavelengths.

The AgBr multilayer lattice spacing became smaller for increasing temperature/lower $\mathrm{RH}$, and the response curve shifted to shorter wavelengths (Blue, B), obeying Bragg's law $(\lambda=2 d \sin \alpha ; \alpha=$ incident angle from sample normal, $d=$ lattice spacing and $\lambda=$ diffraction 


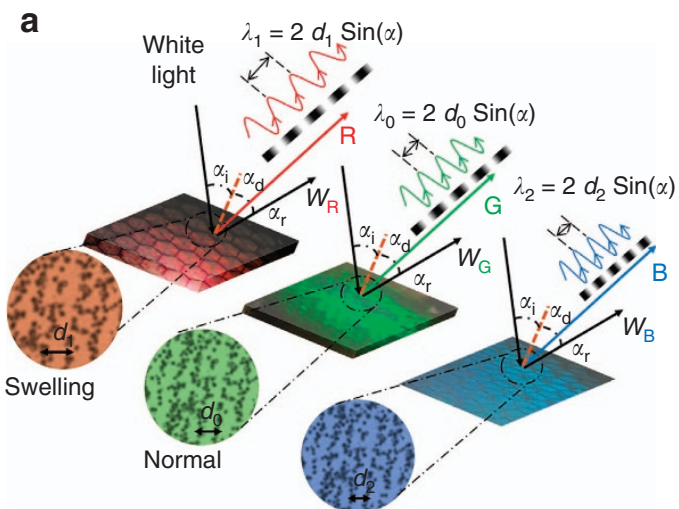

b

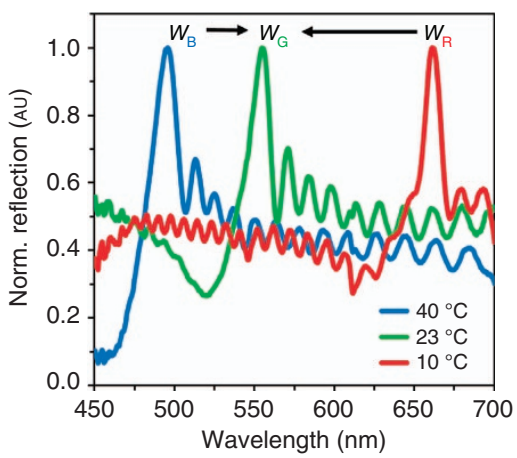

d

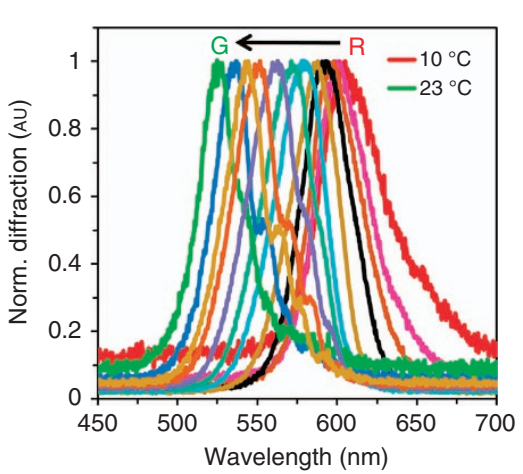

g

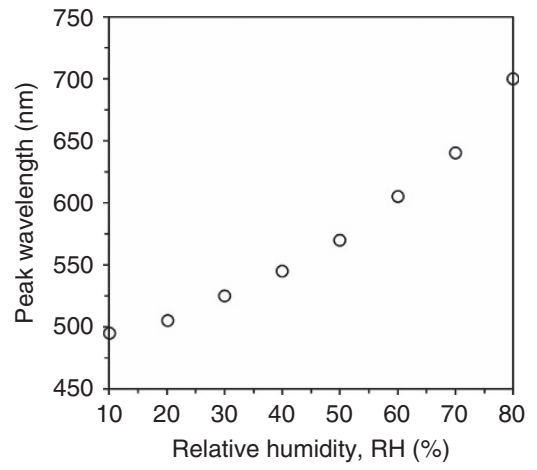

k

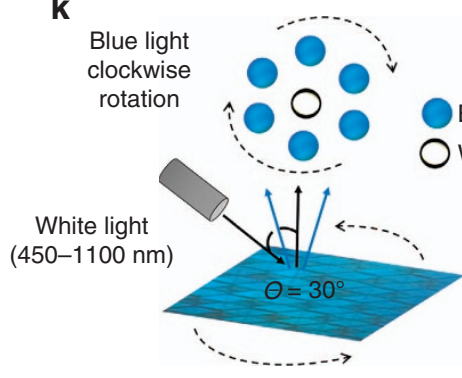

Sample anticlockwise rotation

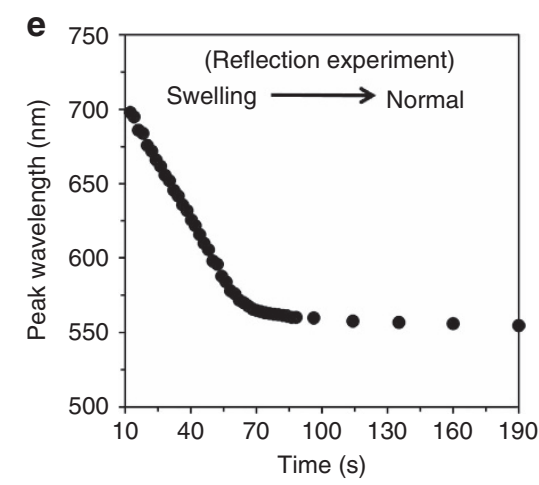

h

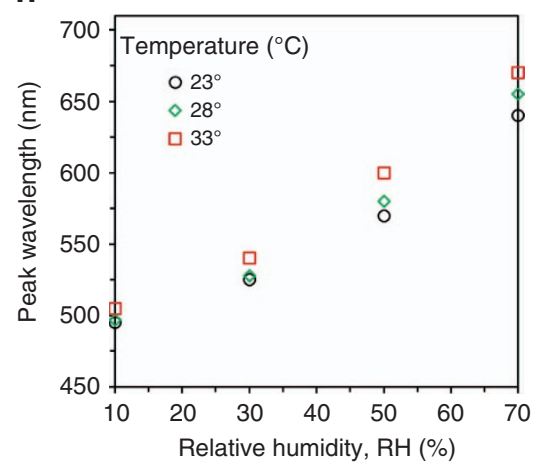

\section{i}
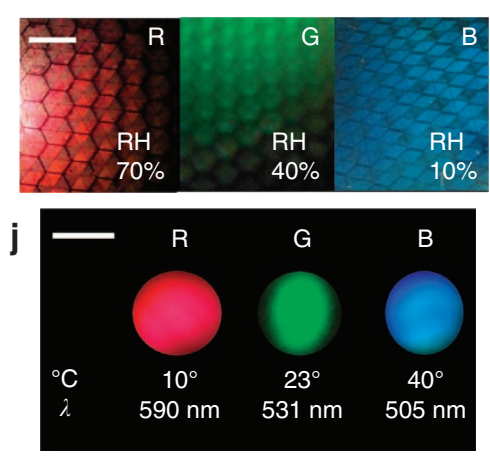

m

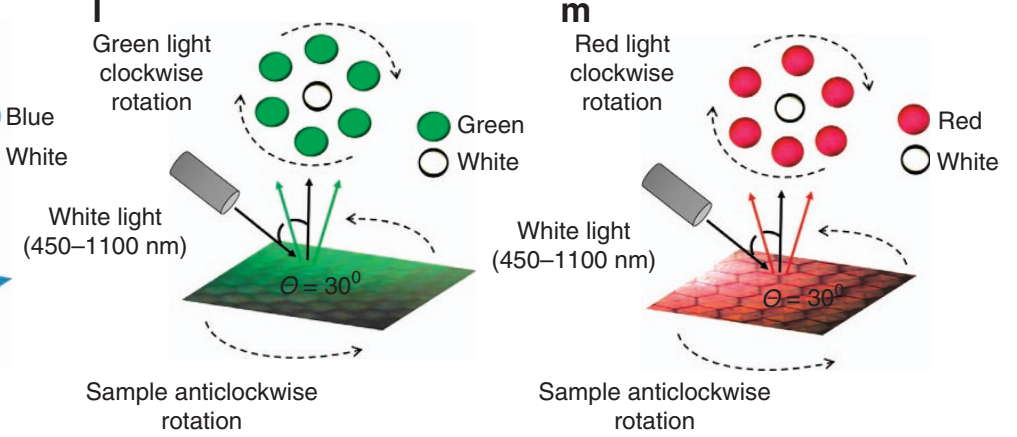

Figure 5 Colorimetric HCCR temperature and RH sensors. (a) Conceptual diagram of the HCCR temperature and RH sensor. The temperature and RH variation act as external stimuli for the sensor, swelling or shrinking the multilayer lattice spacing of the holographic matrix. (b-d) The normalized reflection and diffraction intensity as a function of variation in temperature. White light illumination with incident angle was from $0^{\circ}$ to $30^{\circ}$ during the diffraction experiment. (e, f) Reflected peak wavelength shift as a function of time during swelling/shrinking to initial conditions, respectively (Supplementary Information,Supplementary Movie 3). (g, h) Reflected peak wavelength as a function of RH variation. (i) Holographic color variation with RH variation. Scale bar $=0.4 \mathrm{~cm}$. (j) Far-field diffracted color with temperature variation (Supplementary Information,Supplementary Movie 4). Image projection screen was $30 \mathrm{~cm}$ away from the sensor. Scale bar $=2 \mathrm{~cm}$. (k-m) Rotation property as a function of RH/temperature variation. 
wavelength). Figure 5b-5d shows the reversible HCCR sensor response as a function of temperature variation, which also holds for RH variation. The HCCR tended to retain moisture at low temperatures $\left(10^{\circ} \mathrm{C}\right)$, and the normalized peak wavelength shifted from red to green while shrinking to the normal condition. Similarly, HCCR shrunk at higher temperatures $\left(40^{\circ} \mathrm{C}\right)$ and shifted from blue to green while shrinking to the normal condition. Figure $5 \mathrm{e}$ and $5 \mathrm{f}$ show Bragg peak wavelength shifts as a function of time. Larger Bragg peak shifts were found initially due to higher temperature difference between the holographic polymer and environment. The sensitivity of the HCCR sensor was measured using the gradient of the Bragg peak shift response curve (Supplementary Information 'Temperature and Relative Humidity Sensitivity Measurement'). The sensitivity of the sensor was measured as $\sim 4 \mathrm{~nm}^{\circ} \mathrm{C}^{-1}\left(23^{\circ} \mathrm{C}\right)$ and $1 \mathrm{~nm}(40 \% \mathrm{RH})$.

Figure $5 \mathrm{~g}$ and $5 \mathrm{~h}$ illustrates the HCCR sensor response as a function of $\mathrm{RH}$ variation. The peak wavelength shifted toward longer wavelengths as the RH increased. This was due to the swelling of the recorded HCCR medium and the consequent increase in fringe spacing (Figure $5 \mathrm{~g}$ ). In a real scenario, sensing complexity will be increased when both the temperature and $\mathrm{RH}$ change simultaneously. The peak wavelength shift-dependent function of temperature and $\mathrm{RH}$ variation is

$$
\frac{\Delta \lambda_{\text {Shift }}}{\Delta t}=k \Delta E(T, \mathrm{RH})
$$

where $k$ is the proportionality constant, which is related to the heat transfer coefficient between the recording medium (HCCR matrix) and the environment, and $\Delta E(T, \mathrm{RH})$ is the change in the environment as a function of temperature and $\mathrm{RH}$. The sensor showed a longer wavelength shift with larger RH variation. To evaluate temperature dependence, the peak wavelength shift was measured with RH variation in a controlled environment chamber (Supplementary Information 'Temperature and Relative Humidity Sensitivity Measurement'). The response curve showed (Figure 5h) a small temperature dependence at lower $\mathrm{RH}$ values. A larger wavelength shift occurred due to the increasing temperature and $\mathrm{RH}$ variation. Hence, at higher temperatures, the HCCR matrix expanded by a greater extent for the same RH change. The graphs of wavelength shift vs RH are temperature-dependent; this can be used to sense both temperature and humidity changes. Therefore, the HCCR showed RGB visible color selectivity in response to the temperature or $\mathrm{RH}$ variation that was visible to the naked eye (Figure $5 \mathrm{i}$ ). The far-field sensor readout projection also showed diffracted color change with a variation in temperature (Figure $5 \mathrm{j}$ ). Variation in the external stimuli finely tuned the lattice spacing, which resulted in the Bragg peak in the visible spectrum. The rotational property of HCCR was also tested during the sensing experiments. The rotational property of the HCCR based on the diffraction property was also valid with $\mathrm{RH}$ and temperature variation. The diffracted red, green and blue light (based on $\mathrm{RH}$ and temperature) rotated clockwise, with sample rotation in the anti-clockwise direction (Figure $5 \mathrm{k}-5 \mathrm{~m}$ ). This property of the HCCR sensor is unique, thus offering potential applications in colorimetric sensing and sample tilt/rotation monitoring.

\section{DISCUSSION}

In a conventional CCR array, the directional property is imperative, where an incident light experiences three mirror reflections and returns toward the light source ${ }^{6}$ However, the HCCR array does not produce the three-time reflection effect and return light toward the light source; the light is instead diffracted, as occurs for diffraction gratings and Fresnel lenses, which obey Bragg's law ${ }^{6}$. Hence, unlike the conventional CCRs, one cannot see the reflection of their own eye in the fabricated HCCR plate. However, the rotation property of HCCR based on the diffraction property was demonstrated to characterize the HCCR array. An array of HCCRs was also fabricated without a tilt angle during interference field exposure (Supplementary Information, Supplementary Fig. S5). The holographic CCR array with a tilt angle of $5^{\circ}$ from the surface plane and CCR array with no tilt angle exhibited similar optical effects and spectral readouts.

Practically, distinct properties of the HCCR were found for applications in photonic devices. First, the HCCR array showed wavelength tunability in its diffraction at different incident angles. Hence, it can be used as a visible color filter, wavelength-selective diffraction grating, or printable optical device ${ }^{31,32}$. In addition, the rotational property displayed by the HCCR offers opportunities as a tunable and color-selective mirror in adaptive micro-optics, endoscopic tweezers and wavefront sensing $8,33,34$. The HCCR array can also be used as a security hologram, with its phase conjugation/rotational property being difficult to replicate and easy to verify ${ }^{35-38}$. Such holograms can also be used as wide field of view optical biosensors by fabricating them in functionalized hydrogels, which expand and contract due to changes in analyte concentrations ${ }^{17,19}$.

\section{CONCLUSIONS}

The HCCR array ( $10 \mu \mathrm{m}$ thick) has been developed using a simple top-down fabrication approach. This is the first report of planar CCRs and HCCR arrays produced with silver halide chemistry in Denisyuk reflection mode, which can be used for the highly efficient diffraction/ filtering of particular colors depending on the incident angle. The HCCR array is operated based on Bragg diffraction and exhibited reflection properties and additional wavelength-selective filtering compared with CCRs. The HCCRs exhibited wavefront conjugation, along with tunable wavelength-selective filtering, and acted as a colorimetric temperature and $\mathrm{RH}$ sensor whose readouts were visible to the naked eye. The rotation property is proposed and showed experimentally to characterize the HCCR array. The diffraction intensity of the HCCR array varied with incident angle and wavelength. The presented fabrication approach is simple and allows the production of customized HCCR arrays for different wavelength ranges by changing the recording beam wavelength and exposer tilt angle. Furthermore, the demonstrated approach is also amenable to scaling up using laser manufacturing. The holographic arrays can be recorded using different objects (for example, lenses, diffusers, mirrors), and different polymers can be chosen to diffract at different Bragg peaks or act as a holography sensor. We anticipate that wavelength-selective phase-conjugated HCCRs will lead to applications in diffraction grating-based displays, biosensors and components for photonic integrated circuits.

\section{CONFLICT OF INTEREST}

The authors declare no conflict of interest.

\section{AUTHOR CONTRIBUTIONS}

$\mathrm{HB}$ and $\mathrm{AKY}$ conceived the idea. RA planned and conducted experiments and simulations, analyzed the results, and wrote the article. HB set up the experiment, supervised the experiments and simulations, and led the project. AKY and SHY made intellectual contributions and AKY edited the article.

\section{ACKNOWLEDGEMENTS}

HB thanks the Leverhulme Trust and Wellcome Trust for research funding. We thank Jeff Blyth for discussions. 
1 Yoder PR Jr: Study of Hollow Corner Retroreflectors for Use in a Synchronous Orbit. Danbury, CT: Perkin-Elmer Corp, Optical Technology Div; 1975.

2 Wang XT, He PA, Li S, Wang JY. Test method for single interior right angle in a corner cube prism. Geo-spat Inf Sci 2000; 3: 73-75.

3 Minato A, Sugimoto N. Design of a four-element, hollow-cube corner retroreflector for satellites by use of a genetic algorithm. Appl Opt 1998; 37: 438-442.

4 Ma YF, Li HJ, Lin JP, Yu X, He NL et al. Excellent stability of laser passively Q-switched with a retroreflecting corner cube prism. Laser Phys 2010; 20: 1791-1793.

5 Kaboli M, Farahani HS, Abrishamian MS, Mirtaheri SA. Low-profile, high-gain and broadband array of corner cube reflector antenna based on substrate integrated waveguide. Electron Lett 2011; 47: 363-364.

6 Chipman RA, Shamir J, Caulfield HJ, Zhou QB. Wavefront correcting properties of corner-cube arrays. Appl Opt 1988; 27: 3203-3209.

7 Sobieranski AC, Inci F, Tekin HC, Yuksekkaya M, Comunello E et al. Portable lensless wide-field microscopy imaging platform based on digital inline holography and multiframe pixel super-resolution. Light Sci App/ 2015; 4: e346.

8 Baharav Y, Spektor B, Shamir J, Crowe DG, Rhodes W et al. Wave-front sensing by pseudo-phase-conjugate interferometry. Appl Opt 1995; 34: 108-113.

9 Fainman Y, Lenz E, Shamir J. Contouring by phase conjugation. App/ Opt 1981; 20 158-163.

10 Shamir J. Some Applications for optical phase conjugation. In: Kuhn HJ, Robillard J, editors. Nonlinear Optical Materials. EI Paso, Texas: CRC Press; 1991. 213-222.

11 Chen YF, Yang BJ, Tsai JC. Surface-micromachined MEMS tunable three-leaf trefoiltype corner cube retro-reflector for free-space optical applications. IEEE J Sel Top Quantum Electron 2015; 21: 2700907.

12 Medhat M, Saadany BA, Khalil DA, Mortada B. Opto-mechanical optical path retardation multiplier for optical MEMS applications: US8736843 B2. 2014-05-27.

13 Shaar NS, Barbastathis G, Livermore C. Integrated folding, alignment, and latching for reconfigurable origami microelectromechanical systems. J Microelectromech Syst 2015; 24: 1043-1051.

14 Lee YM, Toda M, Ono T, Esashi M. Miniature interferometer with corner cube mirrors. Proceedings of 2010 IEEE Sensors. IEEE: Kona, HI, 2010.

15 Park JC, Park JY, Won JY, Kim DH, Park J. Silicon bulkmicromachined piezoelectrically actuated corner cube retroflector. Proceedings of the 2011 16th International SolidState Sensors, Actuators and Microsystems Conference (TRANSDUCERS). IEEE: Beijing, 2011.

16 Khalil K, Hassan K, Shebl A, Soliman M, Eltagoury YM et al. MEMS corner-cube transmission-type optical phase modulator in DRIE technology. Proceedings of 2014 International Conference on Optical MEMS and Nanophotonics (OMN). IEEE: Glasgow, 2014.

17 Yetisen AK, Butt H, da Cruz Vasconcellos F, Montelongo Y, Davidson CAB et al. Lightdirected writing of chemically tunable narrow-band holographic sensors. Adv Opt Mater 2014; 2: 250-254.

18 Bjelkhagen HI. Silver-Halide Recording Materials for Holography and Their Processing. Berlin Heidelberg: Springer Science \& Business Media; 1993.

19 Yetisen AK, Montelongo Y, Qasim MM, Butt H, Wilkinson TD et al. Photonic nanosensor for colorimetric detection of metal ions. Anal Chem 2015; 87: 5101-5108.

20 Gurney RW, Mott NF. The theory of the photolysis of silver bromide and the photographic latent image. Proc Roy Soc London A, Math Phys Sci 1938; 164: 151-167.
21 Yetisen AK, Naydenova I, da Cruz Vasconcellos F, Blyth J, Lowe CR. Holographic sensors: three-dimensional analyte-sensitive nanostructures and their applications. Chem Rev 2014; 114: 10654-10696.

22 Ahmed R, Rifat AA, Yetisen AK, Dai Q, Yun SH et al. Multiwall carbon nanotube microcavity arrays. J Appl Phys 2016; 119: 113105.

23 Ahmed R, Rifat AA, Yetisen AK, Salem MS, Yun S-H et al. Optical microring resonator based corrosion sensing. RSC Adv 2016; 6: 56127-56133.

24 Forshaw MRB. Explanation of the "Venetian blind" effect in holography, using the Ewald sphere concept. Opt Commun 1973; 8: 201-206.

25 Butt H, Kidambi PR, Dlubak B, Montelongo Y, Palani A et al. Visible diffraction from graphene and its application in holograms. Adv Opt Mater 2013; 1: 869-874.

26 Butt H, Montelongo Y, Butler T, Rajesekharan R, Dai Q et al. Carbon nanotube based high resolution holograms. Adv Mater 2012; 24: OP331-OP336.

27 Shrestha PK, Chun YT, Chu DP. A high-resolution optically addressed spatial light modulator based on ZnO nanoparticles. Light Sci Appl 2015; 4: e259.

28 Zhang ZC, You Z, Chu DP. Fundamentals of phase-only liquid crystal on silicon (LCOS) devices. Light Sci Appl 2014; 3: e213.

29 Chen WT, Yang KY, Wang CM, Huang YW, Sun G et al. High-efficiency broadband metahologram with polarization-controlled dual images. Nano Lett 2013; 14: 225-230.

30 Ackermann GK, Eichler J. Holography: A Practical Approach. Weinheim, Germany: John Wiley \& Sons. 2008.

31 Zhao QC, Yetisen AK, Anthony CJ, Fowler WR, Yun SH et al. Printable ink holograms Appl Phys Lett 2015; 107: 041115

32 Chen X, Jia BH, Zhang YA, Gu M. Exceeding the limit of plasmonic light trapping in textured screen-printed solar cells using Al nanoparticles and wrinkle-like graphene sheets. Light Sci App/ 2013; 2: e92.

$33 \mathrm{Gu}$ M, Bao HC, Gan XS, Stokes N, Wu JZ. Tweezing and manipulating micro- and nanoparticles by optical nonlinear endoscopy. Light Sci App/ 2014; 3: e126.

34 Sun YL, Dong WF, Niu LG, Jiang T, Liu DX et al. Protein-based soft micro-optics fabricated by femtosecond laser direct writing. Light Sci App/ 2014; 3: e129.

$35 \mathrm{Gu}$ M, Li XP, Cao YY. Optical storage arrays: a perspective for future big data storage. Light Sci Appl 2014; 3: e177.

36 Wen DD, Yue FY, Li GX, Zheng GX, Chan KL et al. Helicity multiplexed broadband metasurface holograms. Nat Commun 2015; 6: 8241.

37 Li XP, Ren HR, Chen X, Liu J, Li Q et al. Athermally photoreduced graphene oxides for three-dimensional holographic images. Nat Commun 2015; 6: 6984.

38 Yetisen AK, Butt H, Mikulchyk T, Ahmed R, Montelongo Y et al. Color-Selective $2.5 \mathrm{D}$ holograms on large-area flexible substrates for sensing and multilevel security. Adv Opt Mater 2016; 4: 1589-1600.

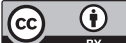

This work is licensed under a Creative Commons Attribution 4.0 International License. The images or other third party material in this article are included in the article's Creative Commons license, unless indicated otherwise in the credit line; if the material is not included under the Creative Commons license, users will need to obtain permission from the license holder to reproduce the material. To view a copy of this license, visit http://creativecommons.org/licenses/by/4.0/

(C) The Author(s) 2017

Supplementary Information for this article can be found on the Light: Science \& Applications' website (http://www.nature.com/lsa) 